\title{
Clinical implications of COVID-19 in patients with chronic liver disease and liver tumor
}

\author{
Martina Gambato ${ }^{1} \cdot$ Patrizia Burra ${ }^{1}$
}

Published online: 5 June 2020

(C) Italian Society of Surgery (SIC) 2020

On March 31st of this year, the World Health Organization (WHO) declared a pandemic infection from severe acute respiratory syndrome coronavirus 2 (SARS-CoV-2), causing 2019 coronavirus disease (COVID-19). As on May 11th, there were around 4 million reported cases worldwide, with more than a quarter of a million deaths.

SARS-CoV-2 is a positive-sense, very long singlestranded RNA virus that shares a part of its genome sequence with SARS-CoV. Like SARS-CoV, it uses angiotensin-converting enzyme 2 (ACE-2) receptor (present in many compartments, including the hepato-biliary tract) to enter the target cells. A preliminary study suggested that ACE-2 receptor is particularly present in cholangiocytes [1], but SARS-CoV-2 infection does not cause bile duct injury, and bile duct markers are not severely altered in infected patients. A liver biopsy from a patient with COVID-19 showed microvesicular liver steatosis, mild lobular and portal activity, a picture resembling drug-induced liver injury (DILI) [2]. Though some drugs used to treat COVID-19 patients are associated with DILI, the altered liver test findings were largely present already on admission, so the DILI hypothesis is weak. The mechanism of liver injury is still unclear, but the most likely one relates to the liver being affected by the immunological reaction and severe inflammation due to the viral infection. Patients with liver injury reveal higher inflammatory markers, such as C-protein reaction, procalcitonin and lymphopenia. They also have fever more frequently, and liver damage is more common in patients with severe pneumonia, which may be associated with an explosion of inflammatory cytokines.

The incidence of elevated alanine aminotransferase and aspartate aminotransferase transaminases associated with

Patrizia Burra

burra@unipd.it

1 Multivisceral Transplant Unit, Gastroenterology, Department of Surgery, Oncology and Gastroenterology, Padua University Hospital, Padua, Italy mild cholestasis in COVID-19 patients ranges from 14 to $53 \%$, peaking within the first two weeks after admission. Whether patients with prior liver disease are more prone to SARS-CoV-2 infection is not yet clear. Patients with liver cirrhosis might be more susceptible to this infection because of their systemic immunocompromised status. According to a recent meta-analysis, the overall prevalence of chronic liver disease among patients with COVID-19 disease was $3 \%$, and the main causes of chronic liver disease were hepatitis $\mathrm{B}$ virus (HBV) or hepatitis $\mathrm{C}$ virus ( $\mathrm{HCV}$ ) infections [3]. The impact of SARS-CoV-2 infection on patients with pre-existing liver conditions, such as chronic viral hepatitis, is even trickier to establish. In patients with chronic liver disease, the addition of a further cause of liver injury-be it virus-directed or immune-mediated-might be expected to impair liver function, especially in the case of advanced liver disease. For instance, experience gained fromSARS-CoV in 2003 showed that concomitant infection with HBV could lead to severe hepatitis. But if the liver damage induced by COVID-19 is immunologically driven, as mentioned earlier, then the immunocompromised status of cirrhotic patients or cancer patients might be more protective than harmful. In a large cohort study on COVID-19 cases in China, 21 individuals $(2.1 \%$ ) had pre-existing $\mathrm{HBV}$, but no data were provided on this specific group's outcome [4]. Extracting data from seven studies, outcomes were not worse in 42 patients with chronic liver disease and COVID-19, as the mortality rate was $0-2 \%$ [5]. A single case of acute chronic liver failure secondary to SARS-CoV-2 infection in a decompensated alcoholic cirrhotic patient was recently reported. The patient responded to empirical antibiotic therapy and supportive care, completely recovering to his prior liver status [6]. Another important point to consider is that some drugs used against COVID-19 are biological agents (e.g. tocilizumab, baricitinib) that might reactivate HBV infection in inactive HBV carriers, or patients with occult HBV, thus causing liver function impairment. Strict monitoring of liver and virological tests are, therefore, warranted and 
antiviral prophylaxis could be indicated in particular clinical settings. Drug-to-drug interactions should be checked before starting COVID-19 treatments, especially in patients with advanced liver disease taking many types of medication, including antiviral agents. A useful tool is available for this purpose: www.covid19-druginteractions.org. In patients with liver injury infected with SARS-CoV-2, hepatotoxic drugs should be used with caution, and liver function should be closely monitored to prevent it from deteriorating. Overall, the reported data are not yet enough for us to know the risk of infection in patients with existing chronic liver disease, or the impact of COVID-19 on their liver status and outcomes. Meanwhile, according to EASL-ESCMID position paper [7], adequate care should be assured-especially for patients with decompensated liver disease-using telemedicine if possible. A Chinese study showed that the risk of SARS-CoV-2 infection and its potential complications in this population can be reduced by applying a simple approach, based on preventive messages and precautions sent to outpatients via WeChat, or adopted for decompensated cirrhotic inpatients [8].

Patients with liver cancer are another special population often coming to the hospital for treatment and monitoring, who may be at higher risk of contracting COVID-19, especially if they are receiving chemotherapy or immunotherapy. The SARS-CoV-2 infection rate in cancer patients treated at a tertiary institution in Wuhan was $0.79 \%$ (12 of 1524 patients), higher than the reported cumulative incidence in the community during the same period [9]. Less than half of these infected patients were being actively treated for their cancer. Even with such small numbers, older patients and those with non-small cell lung carcinoma showed a higher risk of contracting COVID-19. Cancer patients generally had worse outcomes than other patients, with a mortality rate in the range of 5-20\%, liver cancer being under-represented in the current literature [10].

Specific measures for surveillance, follow-up and treatment have been recommended by the EASL, ESMO and ILCA. To ensure an adequate follow-up, outpatient visits could be conducted using telemedicine, whenever feasible, also considering the compliance of patients clearly hampered by their anxiety. Deciding the treatment strategy to avoid cancer progressing beyond the criteria for transplant is more challenging. According to the Society of Surgical Oncology [11], all patients with aggressive hepato-pancreato-biliary malignancies should undergo surgery. In cases where systemic chemotherapy is indicated in addition to surgery, neoadjuvant chemotherapy should be considered with a view to postponing surgery. Still, ablation or embolization should be used for HCC rather than surgical resection, when indicated. While patients with advanced liver cancer underwent locoregional and surgical treatment without any delay at some hospitals with a lower burden of COVID-19, treatment may have been postponed due to the COVID-19 emergency at other hospitals with a heavier burden of COVID-19, as in Lombardy. One group in northern Italy reported that 42 patients were scheduled for HCC treatments (both locoregional and systemic) with a delay of $\geq 2$ months in $26 \%$ of cases in the time of COVID19 (compared with a year before); and in three of these 42 patients locoregional therapies were administered in lieu of previously-planned surgical resections [12]. At our hospital in the Veneto region, oncological strategies have been tailored to the local organization and treatment decisions have been discussed by the multidisciplinary team. Previously-scheduled locoregional treatments were not deferred as a result of the COVID-19 pandemic because we were able to keep beds free for these procedures, and radiological activity went ahead as normal. Considering our hospital resources in the time of COVID-19, we saw a minimal reduction in the surgical procedures for hepatobiliary cancers, as patients with advanced liver cancer or at higher risk of tumor progression underwent liver resection as usual. On the other hand, the number of liver transplants performed between February 21st (when the first case of SARS-CoV-2 was identified in Veneto) and May 4th (the start of the second phase of the SARS-CoV-2 emergency in the region) was lower than in the same period in 2019, given a marked reduction in organ donations in the North Italian Sharing Organ Procurement area.

In conclusion, liver damage during SARS-CoV-2 infection has been reported quite frequently, especially in patients who developed severe COVID-19 disease. Whether the risk of infection and the virus's impact on outcome are worse for patients with existing liver disease is still not entirely clear. Patients with chronic liver disease and liver cancer should, therefore, follow specific measures and precautions to avoid clinical complications related to COVID-19. In COVID-19-saturated institutions, the scarcity of human and hospital resources probably prevented the regular radiological follow-up and oncological treatment of HCC patients. In the near future, our main task will be to establish whether the outbreak has worsened the prognosis for HCC patients, and to promptly reschedule their oncological treatments.

Funding The authors received no financial support to produce this Letter.

\section{Compliance with ethical standards}

Conflict of interest The authors declare no conflict of interest.

Research involving human participants and/or animals This article does not contain any studies with human participants or animals performed by any of the authors. 
Informed consent For this type of study, formal consent is not required.

\section{References}

1. Chai Longfei Hu, Zhang Y, Han W, Zhou Lu, Ke A, Zhou J, Shi G, Fang N, Fan J, Cai J, Fan J, Lan F (2020) Specific ACE2 expression in cholangiocytes may cause liver damage after 2019-nCoV infection. BioRxiv. https://doi.org/10.1101/2020.02.03.931766

2. Xu Z, Shi L, Wang Y, Zhang J, Huang L, Zhang C, Liu S, Zhao P, Liu H, Zhu L, Tai Y, Bai C, Gao T, Song J, Xia P, Dong J, Zhao J, Wang FS (2020) Pathological findings of COVID-19 associated with acute respiratory distress syndrome. Lancet Respir Med $8(4): 420-422$

3. Mantovani A, Beatrice G, Dalbeni A (2020) Coronavirus disease 2019 and prevalence of chronic liver disease: a meta-analysis. Liver Int. https://doi.org/10.1111/liv.14465

4. Guan WJ, Ni ZY, Hu Y et al (2020) Clinical characteristics of coronavirus disease 2019 in China. N Engl J Med 382(18):17081720. https://doi.org/10.1056/NEJMoa2002032

5. Bangash MN, Patel J, Parekh D (2020) COVID-19 and the liver: little cause for concern. Lancet Gastroenterol Hepatol. https://doi. org/10.1016/S2468-1253(20)30084-4

6. Qiu H, Wander P, Bernstein D, Satapathy SK (2020) Acute on chronic liver failure from novel severe acute respiratory syndrome coronavirus 2 (SARS-CoV-2). Liver Int. https://doi.org/10.1111/ liv. 14506

7. Boettler 1, Newsome PN, Mondelli MU, Maticic M, Cordero E, Cornberg M, Berg T (2020) Care of patients with liver disease during the COVID-19 pandemic: EASL-ESCMID position paper. JHEP Rep 2(3):100113

8. Xiao Y1, Pan H2, She Q1, Wang F3, Chen M4 (2020) Prevention of SARS-CoV-2 infection in patients with decompensated cirrhosis. Lancet Gastroenterol Hepatol. https://doi.org/10.1016/ S2468-1253(20)30080-7 (Epub ahead of print)

9. Yu J, Ouyang W, Chua MLK, Xie C (2020) SARS-CoV-2 Transmission in patients with cancer at a Tertiary Care Hospital in Wuhan, China. JAMA Oncol. https://doi.org/10.1001/jamao ncol.2020.0980 (Epub ahead of print)

10. Zhang L, Zhu F, Xie L, Wang C, Wang J, Chen R, Jia P, Guan HQ, Peng L, Chen Y, Peng P et al (2020) Clinical characteristics of COVID-19-infected cancer patients: a retrospective case study in three hospitals within Wuhan. China Ann Oncol. https://doi. org/10.1016/j.annonc.2020.03.296

11. Bartlett DL, Howe JR, Chang G, Crago A, Hogg M, Karakousis G, Levine E, Maker A, Mamounas E, McGuire K et al (2020) Management of cancer surgery cases during the COVID-19 pandemic: considerations. Ann Surg Oncol. 27(6):1717-1720

12. Iavarone M, Sangiovanni A, Carrafiello G, Rossi G, Lampertico P (2020) Management of hepatocellular carcinoma in the time of COVID-19. Ann Oncol. https://doi.org/10.1016/j.annon c.2020.04.007

Publisher's Note Springer Nature remains neutral with regard to jurisdictional claims in published maps and institutional affiliations. 